\title{
White Matter Hyperintensities and Cognitive Dysfunction in Patients With Infratentorial Stroke
}

\author{
Tae Won Kim, MD ${ }^{1}$, Yun-Hee Kim, MD, PhD ${ }^{1,2}$, Kang Hee Kim, MD ${ }^{1}$, Won Hyulk Chang, MD, PhD \\ ${ }^{1}$ Department of Physical and Rehabilitation Medicine, Center for Prevention and Rehabilitation, \\ Heart Vascular and Stroke Institute, Samsung Medical Center, Sungkyunkwan University School of Medicine, Seoul; \\ ${ }^{2}$ Samsung Advanced Institute for Health Science and Technology, Sungkyunkwan University, Seoul, Korea
}

Objective To determine whether cognitive function is associated with white matter hyperintensities (WMH) in patients with infratentorial stroke.

Methods This was a retrospective, cross-sectional study. Twenty-four first-ever infratentorial stroke patients between 18 and 60 years of age were enrolled. WMH was evaluated by the Fazekas scale and the Scheltens scale. Cognitive functions were assessed using the Korean Mini-Mental Status Examination (K-MMSE), Rey-Osterrieth Complex Figure Test, and the Seoul Computerized Neuropsychological Test Battery (SCNT) at one month after stroke. All participants were divided into two groups based on the presence of WMH (no-WMH group and WMH group). General characteristics and cognitive functions were compared between the groups.

Results There were no significant differences in general characteristics, such as age, stroke type, hypertension history, and education level between the two groups. However, K-MMSE in the WMH group was significantly lower compared to the no-WMH group $(\mathrm{p}<0.05)$. The verbal learning test score in SCNT was significantly higher in the no-WMH group compared to the WMH group $(\mathrm{p}<0.05)$. Executive function in the no-WMH group tended to be higher compared to the WMH group.

Conclusion Impairment of cognitive function in patients with infratentorial stroke appeared to be associated with WMH. WMH should be carefully evaluated during rehabilitation of infratentorial stroke patients.

Keywords Leukoaraiosis, Infratentorial, Stroke, Cognition, Leukoencephalopathies

Received April 21, 2014; Accepted July 1, 2014

Corresponding author: Won Hyuk Chang

Department of Physical and Rehabilitation Medicine, Center for Prevention and Rehabilitation, Heart Vascular and Stroke Institute, Samsung Medical Center, Sungkyunkwan University School of Medicine, 50 Irwon-dong, Gangnam-gu, Seoul 135-710, Korea

Tel: +82-2-3410-6068, Fax: +82-2-3410-0052, E-mail: wh.chang@samsung. com

() This is an open-access article distributed under the terms of the Creative Commons Attribution Non-Commercial License (http://creativecommons.org/ licenses/by-nc/3.0) which permits unrestricted noncommercial use, distribution, and reproduction in any medium, provided the original work is properly cited. Copyright () 2014 by Korean Academy of Rehabilitation Medicine 


\section{INTRODUCTION}

White matter changes known as leukoaraiosis are commonly referred to as a periventricular white matter disease, or white matter hyperintensities (WMH), due to their bright white appearance on T2-weighted MRI scans [1]. Cerebral white matter disease is frequently reported on brain imaging, predominantly in older patients [2]. Previously, it was regarded as an incidental finding with no therapeutic consequences. It is now becoming increasingly clear that WMH is associated with cognitive decline especially in attention, executive function, and process speed domains [2,3]. In recent years, due to its apparent clinical relevance, there has been a growing interest in white matter changes, its pathology, epidemiology, risk factors, and treatment options. The pathogenesis of WMH is poorly understood. Hypoxic injury due to hypoperfusion caused by atherosclerosis has been proposed as an etiological factor [4]. This is similar to clinical ischemic stroke or lacunar infarction, especially in the infratentorial lesions [5].

Cognitive impairment in patients with infratentorial stroke is well documented [6-8]. However, mapping the brain lesion with functional loss of certain cognitive domain(s) is still a challenge. Furthermore, the location of the lesions in cerebellar stroke patients was found to have no relevance to cognitive function [6]. The pattern of pathophysiology and cognitive decline are similar in infratentorial stroke and WMH. The differential nature of decline in cognitive function between the infratentorial stroke and a supratentorial WMH remains unclear. WMH may be a suitable model to determine the pathophysiology of cognitive decline in patients with infratentorial stroke.

The purpose of the present study was to determine whether cognitive function is associated with WMH in patients with infratentorial stroke. Therefore, we investigated cognitive function in the patients with infratentorial stroke with and without concomitant WMH.

\section{MATERIALS AND METHODS}

\section{Participants}

This was a retrospective, cross-sectional study with medical chart review. The data were collected from medical records of patients who were admitted to our hospital for infratentorial infarction or hemorrhage. The inclusion criteria for the study were 1) first-ever infratentorial stroke (infarction or hemorrhage), 2) confirmation by CT or MRI, and 3) patients' age between 18 and 60 years. The exclusion criteria were 1) progressive or unstable stroke, 2) pre-existing and active major neurological disease, 3) pre-existing and active major psychiatric disease (such as major depression, schizophrenia, bipolar disease, or dementia), 4) delirium after stroke, and 5) definite hydrocephalus history due to stroke. Delirium after stroke was defined as the Korean Mini-Mental Status Examination (K-MMSE) score <23 [9]. The study protocol was approved by our center's Institutional Review Board (No. 2012-09-034).

Demographic data, a detailed history of premorbid risk factors, and clinical variables that might influence the cognitive function were collected from medical records. Demographic data of stroke patients included age, gender, and years of education. Premorbid stroke risk factors included hypertension, diabetes, and smoking history.

\section{Neuropsychological evaluation}

General cognitive function was measured using the KMMSE [10], Rey-Osterrieth Complex Figure Test (ROCFT) [11], and a Seoul Computerized Neuropsychological Test Battery (SCNT) [12], at 1 month after the stoke onset. SCNT consisted of auditory and visual continuous performance test (CPT) for evaluation of attention, digit span test and verbal learning test for evaluation of language memory, visual span test and visual learning test for evaluation of visuospatial memory, trail making test for evaluation of executive function, and word-color test for evaluation of high cognitive function [12].

\section{WMH measurement}

Each MRI study included fluid-attenuated inversion recovery (FLAIR) images at the acute stroke phase. Visual ratings of WMH were performed by an investigator who was blinded to the clinical details. We applied the simple modified Fazekas rating scale [13] and the more complex Scheltens rating scale [14], except in infratentorial regions which were evaluated by the visual WMH assessment on FLAIR images. The modified Fazekas scale ranges from mild to severe. Mild WMH (grade 1) was defined by punctate lesions in the deep white matter with a maximum diameter of $9 \mathrm{~mm}$ for a single lesion and of 20 

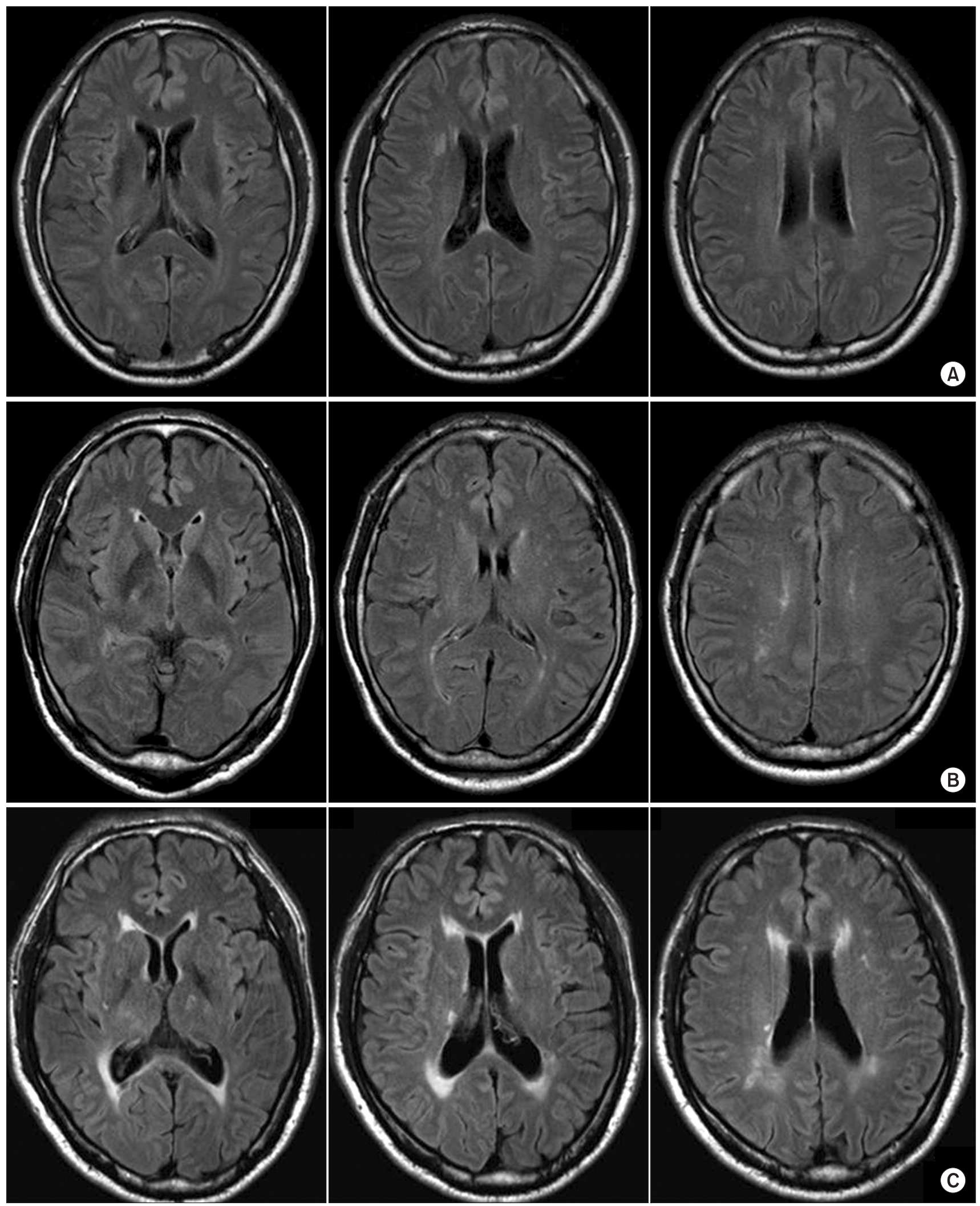

Fig. 1. Brain magnetic resonance imaging according to modified Fazekas scale. (A) No-white matter hyperintensities (WMH) group, (B) mild WMH group (Fazekas grade 1), (C) moderate WMH group (Fazekas grade 2). 
$\mathrm{mm}$ for grouped lesions. Moderate WMH (grade 2) were early confluent lesions of 10-20 $\mathrm{mm}$ single lesions and $>20 \mathrm{~mm}$ grouped lesions in any diameter, and no more than connecting bridges between the individual lesions. Severe WMH (grade 3) were single lesions or confluent areas of hyperintensity of $\geq 20 \mathrm{~mm}$ in any diameter (Fig. 1). The Scheltens scale is a visual rating scale that includes anchored 7-point severity ratings in periventricular, cortical, subcortical, and infratentorial regions [14]. The WMH severity measure for the current study was the sum of ratings for the 3 regions, except for infratentorial region.

\section{Statistical analysis}

Statistical analysis was performed with SPSS ver. 20.0 for Windows (SPSS Inc., Chicago, IL, USA). The KolmogorovSmirnov test was used to assess whether the parametric values were normally distributed. All parametric data were shown to be normally distributed. We compared the values between the two groups (WMH group and no$\mathrm{WMH}$ group) according to the presence of WMH. The WMH group was compared with the reference group by using Student t-test or chi-square test where appropriate. The $\mathrm{p}$-values of less than 0.05 were considered as statistically significant.

\section{RESULTS}

General characteristics and brain images between each group

A total of 24 patients were enrolled in this study, and 12 patients were assigned to each group of WMH and noWMH. There was no significant difference in age, sex, stroke type, or education level between the groups. Also, there was no significant difference in the medical history of hypertension, diabetes, and smoking status between the groups (Table 1).

Six patients had mild WMH and 6 patients had moderate $\mathrm{WMH}$, according to modified Fazekas scale. The score of Scheltens scale in the WMH group was 9.0 5 .9 (Table 2).

\section{Cognitive function between each group}

The mean duration of time from the onset of stroke until the cognitive evaluation was $29.5 \pm 10.9$ days and $27.4 \pm 10.3$ days, with no significant difference between the WMH and no-WMH groups, respectively (Table 3 ).

K-MMSE scores were $26.8 \pm 2.4$ and $29.1 \pm 1.2$ in the $\mathrm{WMH}$ and the no-WMH group, respectively. K-MMSE in the WMH group was significant lower compared to the noWMH group $(\mathrm{p}<0.05)$. However, there was no significant difference in ROCFT between the groups (Table 3).

For visual CPT in the SCNT, the response time in the no-WMH group tended to be shorter compared to the

Table 2. Brain MRI findings in each group

\begin{tabular}{lcc}
\hline & $\begin{array}{c}\text { WMH group } \\
(\mathbf{n}=\mathbf{1 2})\end{array}$ & $\begin{array}{c}\text { No-WMH group } \\
(\mathbf{n}=\mathbf{1 2})\end{array}$ \\
\hline Fazekas scale (0:1:2:3) & $0: 6: 6: 0$ & $0: 0: 0: 0$ \\
Scheltens scale & $9.0 \pm 5.9$ & $0.0 \pm 0.0$ \\
$\begin{array}{l}\text { MRI duration from } \\
\text { onset (day) }\end{array}$ & $10.0 \pm 17.8$ & $4.8 \pm 8.4$ \\
\hline
\end{tabular}

Values are presented as number of patients or mean \pm standard deviation.

MRI, magnetic resonance imaging; $\mathrm{WMH}$, white matter hyperintensities.

Table 1. General characteristics between WMH and no-WMH groups

\begin{tabular}{lccc}
\hline \multicolumn{1}{c}{ Characteristic } & $\begin{array}{c}\text { WMH group } \\
(\mathbf{n = 1 2 )}\end{array}$ & $\begin{array}{c}\text { No-WMH group } \\
(\mathbf{n = 1 2 )}\end{array}$ & p-value \\
\hline Age (yr) & $51.3 \pm 6.4$ & $46.2 \pm 9.8$ & 0.148 \\
\hline Sex (female:male) & $0: 12$ & $2: 10$ & 0.478 \\
Stroke type (infarction:hemorrhage) & $8: 4$ & $11: 1$ & 0.317 \\
Hypertension (yes:no) & $7: 5$ & $4: 8$ & 0.414 \\
Diabetes mellitus (yes:no) & $3: 9$ & $2: 10$ & 1.000 \\
\hline Smoking (pack/yr) & $22.1 \pm 20.4$ & $9.4 \pm 17.3$ & 0.115 \\
Education duration (yr) & $4.1 \pm 1.3$ & $4.5 \pm 1.2$ & 0.534 \\
\hline
\end{tabular}

Values are presented as number of patients or mean \pm standard deviation.

$\mathrm{WMH}$, white matter hyperintensities. 
WMH group, but the difference was not statistically significant. For auditory CPT in the SCNT, the response time was significantly longer in the WMH group compared to the no-WMH group $(\mathrm{p}<0.05)$. The correct response in auditory CPT tended to be lower in the WMH group, but it was not statistically different from the no-WMH group. In the memory domain, the verbal learning test score was significantly lower in the WMH group than in the no-WMH group ( $\mathrm{p}<0.05)$. There was no significant difference in digital span test and visual span test between the groups. Executive function assessed by the trail making test $\mathrm{B}$ in the WMH group tended to be lower compared to the no-WMH group, but the difference was not statistically significant (Table 3 ).

Table 3. Cognitive function between WMH and no-WMH groups

\begin{tabular}{|c|c|c|c|}
\hline & $\begin{array}{c}\text { WMH group } \\
(\mathrm{n}=12)\end{array}$ & $\begin{array}{c}\text { No-WMH group } \\
(n=12)\end{array}$ & p-value \\
\hline Cognitive evaluation duration from onset (day) & $29.5 \pm 10.9$ & $27.4 \pm 10.3$ & 0.761 \\
\hline K-MMSE & $26.8 \pm 2.4^{*}$ & $29.1 \pm 1.2$ & 0.006 \\
\hline \multicolumn{4}{|l|}{ ROCFT } \\
\hline Copy & $28.1 \pm 7.0$ & $27.7 \pm 9.8$ & 0.919 \\
\hline Immediate & $14.7 \pm 8.4$ & $16.9 \pm 8.3$ & 0.564 \\
\hline Delayed & $11.9 \pm 9.5$ & $15.9 \pm 8.9$ & 0.351 \\
\hline \multicolumn{4}{|l|}{ SCNT } \\
\hline \multicolumn{4}{|l|}{ Visual CPT } \\
\hline Correct response & $48.4 \pm 29.3$ & $61.3 \pm 24.0$ & 0.521 \\
\hline Commission error & $62.7 \pm 20.8$ & $77.0 \pm 20.3$ & 0.286 \\
\hline Response time & $32.4 \pm 17.6$ & $42.3 \pm 8.5$ & 0.179 \\
\hline \multicolumn{4}{|l|}{ Auditory CPT } \\
\hline Correct response & $41.1 \pm 21.0$ & $54.1 \pm 14.0$ & 0.088 \\
\hline Commission error & $41.4 \pm 20.2$ & $41.8 \pm 8.5$ & 0.977 \\
\hline Response time & $40.2 \pm 6.8^{*}$ & $48.8 \pm 9.1$ & 0.015 \\
\hline \multicolumn{4}{|l|}{ Digit span } \\
\hline Forward & $41.3 \pm 12.4$ & $48.4 \pm 13.7$ & 0.198 \\
\hline Backward & $43.8 \pm 6.1$ & $48.8 \pm 11.6$ & 0.200 \\
\hline \multicolumn{4}{|l|}{ Visual span } \\
\hline Forward & $42.0 \pm 9.8$ & $45.5 \pm 9.8$ & 0.402 \\
\hline Backward & $40.4 \pm 4.6$ & $44.6 \pm 6.7$ & 0.095 \\
\hline \multicolumn{4}{|l|}{ Verbal learning } \\
\hline Immediate recall & $43.5 \pm 11.4$ & $49.8 \pm 5.7$ & 0.098 \\
\hline Total correct & $33.1 \pm 11.3^{*}$ & $41.5 \pm 8.7$ & 0.048 \\
\hline \multicolumn{4}{|l|}{ Visual learning } \\
\hline Immediate recall & $46.9 \pm 12.2$ & $54.9 \pm 12.8$ & 0.141 \\
\hline Total correct & $50.6 \pm 13.0$ & $54.3 \pm 7.8$ & 0.422 \\
\hline \multicolumn{4}{|l|}{ Trail making } \\
\hline Type A & $45.7 \pm 20.9$ & $47.4 \pm 16.2$ & 0.831 \\
\hline Type B & $38.8 \pm 23.5$ & $43.3 \pm 14.8$ & 0.599 \\
\hline
\end{tabular}

Values are presented as mean \pm standard deviation.

WMH, white matter hyperintensities; K-MMSE, Korean Mini-Mental Status Examination; ROCFT, Rey-Osterrieth Complex Figure Test; SCNT, the Seoul Computerized Neuropsychological Test Battery; CPT, continuous performance test.

${ }^{*} \mathrm{p}<0.05$, between each group. 


\section{DISCUSSION}

This study revealed that cognitive function in the infratentorial stroke patients with WMH was significantly lower compared to with no-WMH. General cognitive function measured by K-MMSE was significantly lower in the infratentorial stroke patients with WMH, compared to no-WMH. Sustained and selective attention assessed by auditory CPT and verbal learning was also lower in the infratentorial stroke patients with WMH. These results suggest that WMH should be considered in the pathophysiology of the cognitive dysfunction, in the infratentorial stroke patients. Therefore, the management for cognitive dysfunction with WMH can be applied for infratentorial stroke patients in the cognitive rehabilitation [4].

The cognitive performance of patients with variously defined white matter changes has been reported in healthy elderly subjects $[15,16]$, in patients with mild cognitive impairment $[17,18]$, and in Alzheimer disease $[19,20]$. The pattern of cognitive deficits in patients with subcortical ischemic lesion is distinctive from that of Alzheimer disease, which resembles Parkinson disease with disproportionate impairment in executive functioning. Severity of white matter changes and diabetes were independent predictors of cognitive decline in an initially nondisabled elderly population [2]. Hypertension, smoking status, diabetes, hyperhomocysteinemia, and heart disease were reported to be the risk factors for white matter changes [21]. Cerebral white matter changes are frequently seen on CT and MR scans of elderly individuals [21]. While the association between WMH and aging has been a consistent finding in various studies, heterogeneous conclusions have been reached with other risk factors [22]. Many discrepancies exist among the results of studies that evaluated the clinical correlates of white matter changes. In this study, the inclusion criterion of age was between 18 and 60 years. Therefore, white matter changes were correlated more with ischemia, rather than with the age of patients in this study [21]. Multiple small vessel infarcts in the subcortical white matter, which are often the result of chronic hypertension, can lead to lipohyalinosis of the small vessels. This process underlies the pathophysiology of white matter changes [4] and is very similar to that of infratentorial stroke [23].

Previous studies have reported the cognitive dysfunc- tion in infratentorial stroke, especially in cerebellar stroke $[6-8,24]$. One report on acquired pediatric hemorrhagic cerebellar lesions showed that neuropsychological impairments were present in all patients [24]. However, in that study, the neuropsychological impairments were mild in the majority of the subjects and were extremely heterogeneous. Also, Alexander et al. [6] reported that damage to some cerebellar sites might have specific cognitive consequences, but the cognitive impairment after focal cerebellar injury in adults was mild or transient. These previous reports suggested that the infratentorial stroke, especially cerebellar lesion might cause mild cognitive dysfunction. However, the definite lesion and pathophysiology that leads to cognitive dysfunction in patients with the infratentorial stroke remain unclarified.

Our results indicated that impairment of cognitive function in patients with infratentorial stroke seemed to be associated with subcortical white matter changes. In order to characterize the cognitive deficits associated with $\mathrm{WMH}$, infratentorial stroke patients were investigated using a detailed neuropsychological battery. The patients with WMH had significantly lower scores in response time of auditory $\mathrm{CPT}$ and verbal learning test, in contrast to the no-WMH group. The performance on neuropsychological test of patients with WMH group was clearly inferior to the no-WMH group, throughout the cognitive function domain. However, these differences were mostly not statistically significant, possibly due to the small sample size. Therefore, the small sample size might be a limitation in this study. We could not describe the results of a comparison analysis between the severity of WMH and the cognitive function, according to the modified Fazekas scale, because of the small sample size. However, our data did reveal subtle differences between the groups, but without the statistical significance.

In conclusion, our study emphasized the impact of white matter changes on attention, memory, and general cognitive function in infratentorial stroke patients. Further investigation and prospective studies are required to determine whether the location of white matter changes increases the risk of cognitive decline and disability in the larger population. Clearly, white matter changes in infratentorial stroke patients should be carefully evaluated in the rehabilitation setting. 


\section{CONFLICT OF INTEREST}

No potential conflict of interest relevant to this article was reported.

\section{ACKNOWLEDGMENTS}

This study was supported by Basic Science Research Program through the National Research Foundation of Korea (NRF) funded by the Ministry of Education, Science and Technology (No. 20110014021).

\section{REFERENCES}

1. Kearney-Schwartz A, Rossignol P, Bracard S, Felblinger J, Fay R, Boivin JM, et al. Vascular structure and function is correlated to cognitive performance and white matter hyperintensities in older hypertensive patients with subjective memory complaints. Stroke 2009;40:1229-36.

2. Verdelho A, Madureira S, Moleiro C, Ferro JM, Santos CO, Erkinjuntti T, et al. White matter changes and diabetes predict cognitive decline in the elderly: the LADIS study. Neurology 2010;75:160-7.

3. Debette S, Markus HS. The clinical importance of white matter hyperintensities on brain magnetic resonance imaging: systematic review and meta-analysis. BMJ 2010;341:c3666.

4. Roman GC, Erkinjuntti T, Wallin A, Pantoni L, Chui HC. Subcortical ischaemic vascular dementia. Lancet Neurol 2002;1:426-36.

5. Cereda C, Carrera E. Posterior cerebral artery territory infarctions. Front Neurol Neurosci 2012;30:128-31.

6. Alexander MP, Gillingham S, Schweizer T, Stuss DT. Cognitive impairments due to focal cerebellar injuries in adults. Cortex 2012;48:980-90.

7. Nys GM, van Zandvoort MJ, de Kort PL, Jansen BP, de Haan EH, Kappelle LJ. Cognitive disorders in acute stroke: prevalence and clinical determinants. Cerebrovasc Dis 2007;23:408-16.

8. Frank B, Schoch B, Hein-Kropp C, Dimitrova A, Hovel $\mathrm{M}$, Ziegler $\mathrm{W}$, et al. Verb generation in children and adolescents with acute cerebellar lesions. Neuropsychologia 2007;45:977-88.

9. Mori E, Yamadori A. Acute confusional state and acute agitated delirium: occurrence after infarction in the right middle cerebral artery territory. Arch Neurol 1987;44:1139-43.

10. Folstein MF, Folstein SE, McHugh PR. "Mini-mental state": a practical method for grading the cognitive state of patients for the clinician. J Psychiatr Res 1975;12:189-98.

11. Somerville J, Tremont G, Stern RA. The Boston Qualitative Scoring System as a measure of executive functioning in Rey-Osterrieth Complex Figure performance. J Clin Exp Neuropsychol 2000;22:613-21.

12. Kim YH, Shin SH, Park SH, Ko MH. Cognitive assessment for patient with brain injury by computerized neuropsychological test. J Korean Acad Rehabil Med 2001;25:209-16.

13. Pantoni L, Basile AM, Pracucci G, Asplund K, Bogousslavsky J, Chabriat $\mathrm{H}$, et al. Impact of age-related cerebral white matter changes on the transition to disability: the LADIS study: rationale, design and methodology. Neuroepidemiology 2005;24:51-62.

14. Scheltens P, Barkhof F, Leys D, Pruvo JP, Nauta JJ, Vermersch $\mathrm{P}$, et al. A semiquantative rating scale for the assessment of signal hyperintensities on magnetic resonance imaging. J Neurol Sci 1993;114:7-12.

15. Kramer JH, Mungas D, Reed BR, Schuff N, Weiner MW, Miller BL, et al. Forgetting in dementia with and without subcortical lacunes. Clin Neuropsychol 2004;18:32-40.

16. Kramer JH, Reed BR, Mungas D, Weiner MW, Chui HC. Executive dysfunction in subcortical ischaemic vascular disease. J Neurol Neurosurg Psychiatry 2002;72:217-20.

17. Galluzzi S, Sheu CF, Zanetti O, Frisoni GB. Distinctive clinical features of mild cognitive impairment with subcortical cerebrovascular disease. Dement Geriatr Cogn Disord 2005;19:196-203.

18. de Mendonca A, Ribeiro F, Guerreiro M, Palma T, Garcia C. Clinical significance of subcortical vascular disease in patients with mild cognitive impairment. Eur J Neurol 2005;12:125-30.

19. Schmidtke K, Hull M. Neuropsychological differentiation of small vessel disease, Alzheimer's disease and mixed dementia. J Neurol Sci 2002;203-204:17-22.

20. Tierney MC, Black SE, Szalai JP, Snow WG, Fisher RH, Nadon G, et al. Recognition memory and verbal fluency differentiate probable Alzheimer disease from subcortical ischemic vascular dementia. Arch Neurol 
2001;58:1654-9.

21. Pantoni L. Pathophysiology of age-related cerebral white matter changes. Cerebrovasc Dis 2002;13 Suppl 2:7-10.

22. Pantoni L, Garcia JH. The significance of cerebral white matter abnormalities 100 years after Binswanger's report: a review. Stroke 1995;26:1293-301.

23. De Girolami U, Seilhean D, Hauw JJ. Neuropathology of central nervous system arterial syndromes. Part I: the supratentorial circulation. J Neuropathol Exp Neurol 2009;68:113-24.

24. Wingeier K, Bigi S, El-Koussy M, Heinks-Maldonado T, Boltshauser E, Steinlin M. Long-term sequelae after acquired pediatric hemorrhagic cerebellar lesions. Childs Nerv Syst 2011;27:923-31. 